\title{
Autologous Anti-BCMA-CAR Expressing Stem Memory T-cells P-BCMA-101
}

National Cancer Institute

\section{Source}

National Cancer Institute. Autologous Anti-BCMA-CAR Expressing Stem Memory T-cells

P-BCMA-101. NCI Thesaurus. Code C142864.

A preparation consisting of autologous T-cells that are enriched to be primarily stem memory T-cells (Tscm) and are transfected by electroporation with a proprietary transposon-based DNA plasmid vector (PiggyBac) containing an undisclosed selection gene and encoding both an unidentified human-derived safety switch and a chimeric antigen receptor (CAR) based on a proprietary non-immunog lobulin scaffold molecule Centyrin (CART yrin), which specifically recognizes human B-cell maturation antigen (BCMA; tumor necrosis factor receptor superfamily member 17; TNFRSF17), with potential immunostimulating and antineoplastic activities. Upon administration, autologous anti-BCMA-CAR-expressing Tscm P-BCMA-101 specifically recognize and induce selective toxicity in BCMA-expressing tumor cells. Use of CART yrin may elicit less immunotoxicity than agents based on antibody-derived single chain variable fragments (scFv), and this agent may exhibit increased persistence and decreased exhaustion for the administered T-cells. If significant side effects occur, the safety switch mechanism can induce the rapid attenuation or elimination of P-BCMA-101. BCMA, a tumor-specific antigen and a member of the tumor necrosis factor receptor superfamily (TNFRSF) that binds to both a proliferation-inducing ligand (APRIL; TNFSF13) and B-cell activating factor (BAFF; TNFSF13B), plays a key role in plasma cell survival. BCMA is found on the surfaces of plasma cells and is overexpressed on malignant plasma cells. 